\title{
Síndrome colestásico en una paciente con esferocitosis
}

\section{hereditaria}

\section{Cholestasis syndrome in a patient with hereditary spherocytosis}

1 Yessenia Cruz Castillo

http://orcid.org/0000-0002-4642-885X Médico Especialista en Medicina Interna del Ecuador, (Tungurahua/Ambato) Médico Internista del Hospital Regional Docente Ambato, Docente Universidad Técnica de Ambato (UTA). ym.cruz@uta.edu.ec

$2 \quad$ Juan Fernando Orozco Herrera https://orcid.org/0000-0002-3916-2985 Médico Especialista en Medicina Interna del Ecuador, (Tungurahua/Ambato) Médico Internista del Hospital Regional Docente Ambato, Universidad Regional Autónoma de los Andes "Uniandes" ua.juanorozco@uniandes.edu.ec

$3 \quad$ Viviana Lizbeth Naranjo Aldas https://orcid.org/0000-0001-5259-2405 Externa de la Carrera de Medicina, Facultad de Ciencias de la Salud, Universidad Técnica de Ambato (UTA), Tungurahua, Ecuador) vnaranjo4859@uta.edu.ec

4 Johnny Steven Valdez Benalcazar https://orcid.org/0000-0002-0766-4702 Médico Cirujano en Universidad Regional Autónoma de los Andes "Uniandes", Tungurahua, Ambato ma.johnnysvb29@uniandes.edu.ec

Artículo de Investigación Científica y Tecnológica Enviado: 05/01/2022 Revisado: $15 / 01 / 2022$ Aceptado: 14/02/2022 Publicado:24/02/2022 DOI: https://doi.org/10.33262/anatomiadigital.v5i1.2075 
Palabras claves:

esferocitosis, anemia, colelitiasis $\mathrm{y}$ colecistectomía
Resumen

Introducción: Entre las anemias hemolíticas de carácter hereditario, la esferocitosis hereditaria (HS) es aquella que se presenta con mayor frecuencia. Los patrones de herencia autosómico dominante (AD) y autosómico recesivo (AR) representan el $75 \%$ y el $25 \%$ de todos los casos de HS, respectivamente. La prevalencia de la HS varía entre las diferentes regiones raciales y étnicas, y afecta aproximadamente a 1 de cada 2000 personas en el norte de Europa, América del Norte y Japón, pero es menos común en los afroamericanos y en el sudeste asiático. El síndrome colestásico es una de las complicaciones más comunes y suele presentarse en pacientes entre 10 y 30 años, aunque los pacientes más jóvenes también pueden presentarlo y la colecistectomía es un tratamiento radical seguro y eficaz para esta patología. Objetivo: Describir la evolución clínica y el manejo quirúrgico de la paciente con esferocitosis que desarrolla un síndrome colestásico como una de las complicaciones más frecuentes de esta patología. Materiales y métodos: El sustento teórico de este caso clínico, correspondiente a la información más actualizada hasta 5 años atrás, se obtuvo a través de la búsqueda de literatura y el acceso a artículos científicos, libros, revistas y otros documentos físicos y virtuales a través de buscadores profesionales en el campo de la salud. Conclusiones: La evolución de las complicaciones de la esferocitosis hereditaria en especial del síndrome colestásico conllevo a varios días de estancia hospitalaria, pero en la mayoría de las ocasiones se suelen resolver favorablemente en este caso por medio de la colecistectomía laparoscópica se obtuvo buenos resultados y un alta a los 22 días desde su ingreso.

\section{Keywords:}

Spherocytosis, anemia, cholelithiasis and cholecystectomy

\section{Abstract}

Introduction: Among hereditary hemolytic anemias, hereditary spherocytosis (HS) is the one that occurs most frequently. Autosomal dominant (AD) and autosomal recessive (AR) inheritance patterns account for $75 \%$ and $25 \%$ of all HS cases, respectively. The prevalence of HS varies among different racial and ethnic regions, affecting approximately 1 in 2,000 people in Northern Europe, North America, and Japan, but is less common in African Americans and Southeast Asia. Cholestatic syndrome is usually one of the most common complications and occurs in 
patients between 10 and 30 years of age, although younger patients can also present it and cholecystectomy is a safe and effective radical treatment for this pathology. Objective: To describe the clinical evolution and the surgical management of the patient with spherocytosis who develops a cholestatic syndrome as one of the most frequent complications of this pathology. Materials and methods: The theoretical support of this clinical case, corresponding to the most updated information up to 5 years ago, was obtained through the literature search and access to scientific articles, books, magazines, and other physical and virtual documents through of professional seekers in the field of health. Conclusions: The evolution of the complications of hereditary spherocytosis, especially cholestatic syndrome, led to several days of hospital stay, but in most cases, they are usually resolved favorably in this case by means of laparoscopic cholecystectomy, satisfactory results were obtained and discharge. 22 days after admission.

\section{Introducción}

Entre las anemias hemolíticas de carácter hereditario, la esferocitosis hereditaria (HS) es aquella que se presenta con mayor frecuencia. Aproximadamente el 20-30\% de los pacientes con HS son asintomáticos, por lo que el diagnóstico inicial puede retrasarse hasta la edad adulta $(1,2)$. En la HS los eritrocitos no pueden mantener su forma bicóncava normal debido a mutaciones genéticas en las proteínas de la membrana / citoesqueleto que juegan un papel en la estabilidad morfológica estructural y se hereda principalmente de forma autosómica dominante (2).

Las manifestaciones clínicas varían según la gravedad de la enfermedad, habitualmente existe heterogeneidad en la sintomatología de los pacientes que padecen los diferentes tipos de HS. Los síntomas típicos de HS incluyen anemia, ictericia y esplenomegalia $(3,4)$. Sin embargo, los pacientes con anemia como talasemia (THAL) y anemia hemolítica autoinmune (AIHA) también presentan anemia, ictericia y esplenomegalia $(3,5,6)$. El cuadro clínico de la HS (anemia, ictericia y esplenomegalia) y una complicación común (colelitiasis) son similares a las de los pacientes con anemia como THAL y AIHA. Por estas razones, es complicado discernir la HS de otros patrones anémicos $(7,8,9)$. 
La HS generalmente se diagnostica sobre la base de antecedentes familiares positivos, esferocitosis, ictericia y esplenomegalia. El fenotipo HS también puede modificarse por la coexistencia de otros trastornos como la glucosa-6-fosfato deshidrogenasa (G6PD) y el síndrome de Gilbert $(8,10,11)$. La adaptación de la secuenciación de próxima generación (NGS) ha conducido a un avance asombroso en el diagnóstico de desórdenes genéticos y ha proporcionado beneficios sin precedentes tanto para la medicina de laboratorio personalizada como para los pacientes con trastornos genéticos raros. NGS ha promovido el diagnóstico molecular de HS en comparación con la práctica clínica, incluido tanto el panel dirigido como la secuenciación del exoma completo (WES). Es por eso por lo que las pruebas de diagnóstico de uso común para la HS no tienen una sensibilidad o especificidad ideales. Por lo tanto, algunos pacientes con HS son fácilmente diagnosticados erróneamente o pasados por alto $(3,5,12,13,14)$.

Por otro lado, los analizadores de hematología se utilizan ampliamente en los laboratorios de los hospitales debido a sus procedimientos de control de calidad y rendimiento altamente automatizados, y algunos parámetros de las células sanguíneas pueden ser adecuados para la detección temprana de HS $(3,4)$. La concentración media de hemoglobina corpuscular (MCHC) se incluyó como uno de los criterios de diagnóstico de HS en la guía para el diagnóstico y manejo de HS en 2004, y también se ha informado que el volumen corpuscular esférico medio (MSCV) tiene valor diagnóstico en HS. Además, recientemente se ha encontrado que el volumen de reticulocitos media (MRV) es un índice valioso para el diagnóstico de HS $(8,13,15)$.

La composición de cálculos de bilirrubinato que en un futuro conducirán a un síndrome colestásico que es una de las complicaciones más frecuentes de HS y la primera y tercera década de la vida son las etapas en las que los casos son diagnosticados. Se debe hacer énfasis en que el riesgo se incrementa 5 veces en aquellos individuos que presentan coherencia con Síndrome de Gilbert $(2,7,8,15)$.

Por lo previamente mencionado, se expone el caso clínico de una paciente femenina de 22 años que presenta dolor abdominal a nivel de epigastrio de gran intensidad 8/10 en escala de EVA, que se irradia a hipocondrios y sin causa aparente, se acompaña de nausea que llega al vomito por 5 ocasiones de contenido alimenticio y una deposición liquida. Como antecedente patológico personal relevante el diagnostico de anemia hemolítica (esferocitosis hereditaria) hace 1 año y 2 meses en tratamiento con ácido fólico. Esta paciente se le evaluó y se realiza seguimiento en el Hospital General Docente Ambato.

\section{Descripción del caso}

Paciente femenina de 22 años de raza mestiza acude al Servicio de Urgencias de un Hospital General, con antecedente de esferocirosis hereditaria desde la infancia, por presentar cuadro de 36 horas de evolución por cuadro de dolor abdominal tipo cólico 
localizado en epigastrio e hipocondrio derecho, acompañado de náusea que llega al vómito por varias ocasiones, coluria y notando colocación amarillenta a nivel de piel y conjuntivas. Tiene antecedente familiar por madre de anemia hemolítica. Durante su infancia y adolescencia ha presentado varias crisis hemolíticas, sin embargo, en últimos 3 años ha permanecido estable sin precisar transfusiones y requiriendo ácido fólico de forma permanente. En la exploración destaca la ictericia marcada mucocutánea, esplenomegalia con $6 \mathrm{~cm}$ por debajo del reborde costa, con dolor en la palpación en epigastrio e hipocondrio derecho, con Murphy positivo. Por lo que se cataloga como cuadro colestásico de etiología mixta (hemólisis + cuadro obstructivo biliar) junto con una crisis hemolítica severa y posible hepatitis. Se realizan pruebas complementarias, en las que destacan una anemia normocítica grave más un patrón colestásico marcado (tabla 1). Se realiza una ecografía abdominal se evidencia signos de colecistitis con engrosamiento de la pared vesicular, distendida y líquido perivesicular, además colodocó dilatado con presencia de lito en su interior (figura 1). Se decide colocar terapia antibiótica a base de ampicilina sulbactam y ácido ursodesoxicólico, además se realiza una conlangiopancreatografía retrógrada (CPRE) donde reportan un colédoco dilatado con presencia en el conducto hepático común imagen compatible con cálculo, realizan extracción de este (figura 2). Evoluciona favorablemente con descenso del patrón colestásico. A sus 7 días de estancia hospitalaria realizan resolución quirúrgica por colecistectomía en donde se encuentra vesícula distendida de paredes gruesas intrahepáticas, con múltiples cálculos pequeños en moderada cantidad, con liquido inflamatorio perivesicular. Mantuvo evolución clínica sin complicaciones, recibiendo a su alta ácido fólico.

Tabla 1. Exámenes complementarios: biometría hemática y química sanguínea

\begin{tabular}{ccc}
\hline Parámetros & Valores del paciente & Unidades \\
\hline Leucocitos & $13.220 \mathrm{~K} / \mathrm{ul}^{*}$ & $\mathrm{~K} / \mathrm{ul}$ \\
Hematíes & $2.45 \mathrm{M} / \mathrm{ul}^{*}$ & $\mathrm{M} / \mathrm{ul}$ \\
Hemoglobina & $4.7 \mathrm{~g} / \mathrm{dL}^{*}$ & $\mathrm{~g} / \mathrm{dL}$ \\
Hematocrito & $15.4 \% *$ & $\%$ \\
Volumen corpuscular & $91.6 \mathrm{fL}$ & $\mathrm{fL}$ \\
medio & $31.3 \mathrm{pg}^{*}$ & $\mathrm{Pg}$ \\
$\begin{array}{c}\text { Hemoglobina } \\
\text { corpuscular media }\end{array}$ & &
\end{tabular}


Tabla 1. Exámenes complementarios: biometría hemática y química sanguínea (continuación)

\begin{tabular}{|c|c|c|}
\hline Parámetros & Valores del paciente & Unidades \\
\hline $\begin{array}{l}\text { Concentración de } \\
\text { hemoglobina } \\
\text { corpuscular media }\end{array}$ & $34.2 \mathrm{~g} / \mathrm{dL}$ & $\mathrm{g} / \mathrm{dL}$ \\
\hline Plaquetas & $213 \mathrm{~K} / \mathrm{ul}$ & $\mathrm{K} / \mathrm{ul}$ \\
\hline RDW - SD & $100.9 \mathrm{fL} *$ & $\mathrm{fL}$ \\
\hline $\mathrm{RDW}-\mathrm{CV}$ & $31.4 \% *$ & $\%$ \\
\hline Neutrófilos & $90.0 \% *$ & $\%$ \\
\hline Linfocitos & $7.3 \% *$ & $\%$ \\
\hline Monocitos & $2.5 \%$ & $\%$ \\
\hline Eosinófilos & $0.0 \% *$ & $\%$ \\
\hline Basófilos & $0.2 \%$ & $\%$ \\
\hline Glucosa & $108 \mathrm{mg} / \mathrm{dL}$ & $\mathrm{mg} / \mathrm{dL}$ \\
\hline Creatinina & $0.71 \mathrm{mg} / \mathrm{L}$ & $\mathrm{mg} / \mathrm{L}$ \\
\hline Bilirrubina total & $65.7 \mathrm{mg} / \mathrm{dL}$ & $\mathrm{mg} / \mathrm{dL}$ \\
\hline Bilirrubina directa & $30.00 \mathrm{mg} / \mathrm{dL}^{*}$ & $\mathrm{mg} / \mathrm{dL}$ \\
\hline Bilirrubina indirecta & $35.70 \mathrm{mg} / \mathrm{dL}^{*}$ & $\mathrm{mg} / \mathrm{dL}$ \\
\hline $\mathrm{AST}-\mathrm{TGO}$ & $185 \mathrm{U} / 1^{*}$ & $\mathrm{U} / \mathrm{l}$ \\
\hline $\mathrm{ALT}-\mathrm{TGP}$ & $298 \mathrm{U} / 1^{*}$ & $\mathrm{U} / 1$ \\
\hline Gamma GT & $543 \mathrm{U} / 1^{*}$ & $\mathrm{U} / 1$ \\
\hline Fosfatasa Alcalina & $222 \mathrm{U} / \mathrm{l}^{*}$ & $\mathrm{U} / 1$ \\
\hline Amilasa & $6 \mathrm{U} / /^{*}$ & $\mathrm{U} / \mathrm{l}$ \\
\hline Lipasa & $8 \mathrm{U} / 1$ & $\mathrm{U} / 1$ \\
\hline
\end{tabular}



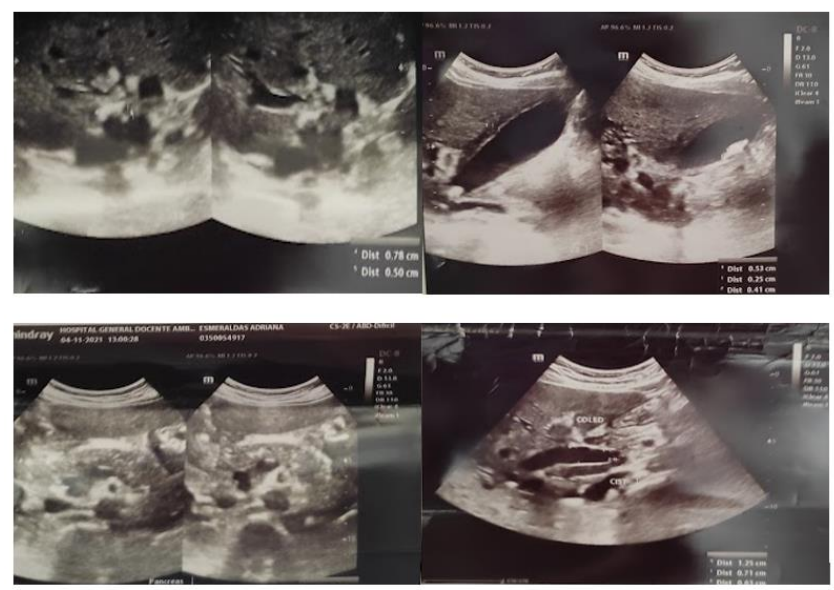

Figura 1. Ecografía abdominal. Nota: se evidencia vía biliar dilatada, en el colédoco se evidencia imagen compatible con lito que mide 9.3 x $4.5 \mathrm{~mm}$. Vesícula biliar distendida 10 x $3.3 \mathrm{~cm}$, con pared engrosada de hasta $6 \mathrm{~mm}$, con edema perivesicular, en su interior varios litos móviles de entre 2 y $6 \mathrm{~mm}$, así como algunos litos fijos a nivel del cuello vesicular entre 5 y 7.5. Fuente: Imagenología del Hospital Docente Ambato
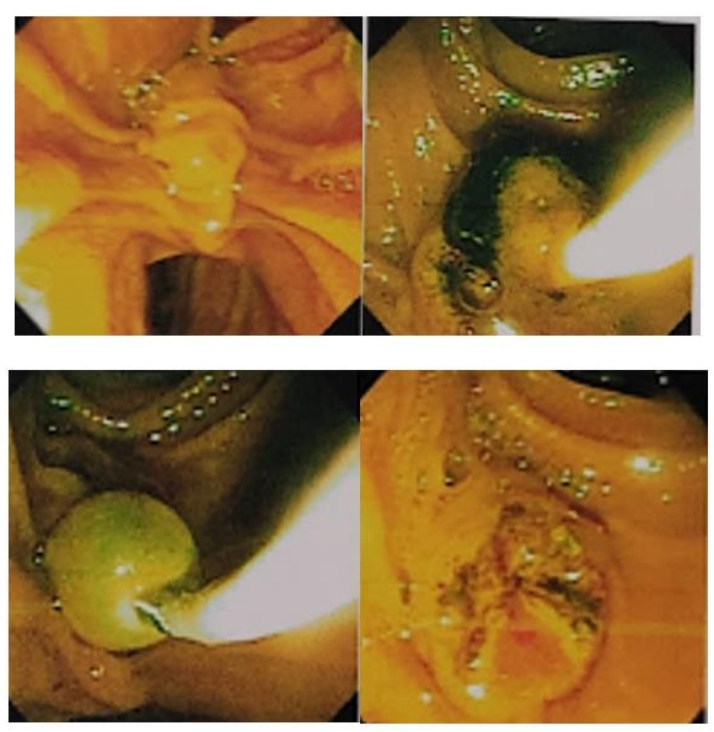

Figura 2. CPRE: Se observa papila edematosa, larga en la segunda porción, ostium cerrado de difícil canulación. En la vía biliar los intrahepáticos normales, el colédoco mida $15 \mathrm{~mm}$ de diámetro a nivel del hepático común se observa una imagen radiológica compatible con un cálculo y el cístico de inserción baja. Fuente: Centro especializado de endoscopia y vía biliar.

\section{Discusión}

La esferocitosis hereditaria (HS) es una anemia hemolítica hereditaria causada por mutaciones en los genes que codifican las proteínas de la membrana de los eritrocitos y se hereda principalmente de forma autosómica dominante (2). Cuando se diagnostica demasiado tarde, la HS conlleva el riesgo de complicaciones a largo plazo, como cálculos 
biliares y anemia grave. El síndrome colestásico es una de las complicaciones más comunes y suele presentarse en pacientes entre 10 y 30 años, aunque los pacientes más jóvenes también pueden presentarlo (15).

En este caso la paciente femenina de 22 años con antecedentes patológicos familiares por parte de su madre con esferocitosis hereditaria que es un trastorno autosómico dominante producto de trastornos de la membrana eritrocitaria y con antecedentes patológicos personales de esferocitosis hereditaria desde la infancia. Ingresó a esta casa de salud el 03 de noviembre del 2021 por desarrollar una complicación muy frecuente de esta patología conocida como síndrome colestásico, el cual presenta la siguiente sintomatología dolor en epigastrio que se irradia a hipocondrio derecho de moderado intensidad 7/10 en escala de EVA, nauseas que llegan al vomito por 5 ocasiones y 1 deposición liquida. En el examen físico se evidencia piel ictérica, escleras ictéricas, abdomen suave, depresible, doloroso a la palpación en hipocondrio derecho, Murphy positivo y ruidos hidroaéreos disminuidos.

En su estancia hospitalaria se le pidieron exámenes de laboratorio que revelan un síndrome anémico, con elevación de los leucocitos y neutrófilos, elevación de todo el perfil hepático (tabla 1). En los exámenes de imagen realizados en esta unidad reportaron una ecografía de abdomen (figura 1) en donde la vía biliar se encuentra dilatada, en el colédoco se evidencia imagen compatible con lito que mide $9.3 \times 4.5 \mathrm{~mm}$, vesícula biliar distendida $10 \times 3.3 \mathrm{~cm}$, con pared engrosada de hasta $6 \mathrm{~mm}$, con edema perivesicular, en su interior varios litos móviles de entre 2 y $6 \mathrm{~mm}$, así como algunos litos fijos a nivel del cuello vesicular entre 5 y 7.5. En la tomografía axial computarizada se reportó la presencia de lito en la vía biliar (colédoco) y esplenomegalia. Determinando que el cuadro de colestasis es secundario a una obstrucción de la vía biliar por un lito.

Una vez analizados estos hallazgos fue necesario realizar una CPRE, la misma que nos reveló una vía biliar dilatada (colédoco $15 \mathrm{~mm}$ ), en el conducto hepático común se observa una imagen radiológica de efecto compatible con un cálculo, el mismo que es extraído en ese momento (figura 2), resolviendo de esta manera el cuadro de colestasis.

Con estos resultados se interconsultó al servicio de cirugía los mismos que decidieron realizar COLELAP a los 18 días de hospitalización, la misma que reportó una vesícula de paredes gruesas intrahepáticas con varios cálculos pequeños en moderada cantidad más liquido inflamatorio perivesicular y finalmente el cuadro evolucionó sin complicaciones. Se dio la resolución y alta del caso a los 22 días de estancia hospitalaria con un posterior control de la patología de base (HS) por consulta externa.

\section{Conclusión}

- La evolución de las complicaciones de la esferocitosis hereditaria en especial del 


\section{ADÉnigital}

síndrome colestásico conllevo a varios días de estancia hospitalaria, pero en la mayoría de las ocasiones se suelen resolver favorablemente en este caso por medio de la colecistectomía laparoscópica se obtuvo buenos resultados y un alta a los 22 días desde su ingreso.

- Los cálculos biliares que son producto de un exceso de bilirrubina, cuya causa es la constante degradación de los glóbulos rojos se forman en la vesícula y pasarán de esta al conducto cístico o al conducto biliar común y en cualquiera de estos lugares pueden quedarse impactados desarrollando así un síndrome colestásico en donde se produce una colecistitis por inflamación de la vesícula biliar una coledocolitiasis si el impacto fue a nivel colédoco y una colangitis por obstrucción de la vía biliar y la consiguiente ascensión de las bacterias a los conductos biliares, desencadenando así toda la sintomatología clásica de esta patología.

- Las manifestaciones clínicas del síndrome colestásico que se presentaron en esta paciente como el dolor a la palpación en epigastrio que se irradia a hipocondrio, nauseas que llegan al vomito, deposiciones liquidas, Murphy positivo y la disminución de los ruidos hidroaéreos; son pilares fundamentales para pensar en esta patología.

- Los exámenes complementarios que se realizaron ante indicios de un síndrome colestásico son la ecografía de abdomen, tomografía axial computarizada de abdomen y la colangiopancreatografia retrograda endoscópica, los mismos que reportaron en su totalidad la existencia de un síndrome colestásico y a su vez la CPRE sirvió como un medio de resolución quirúrgica porque se pudo extraer los cálculos por medio de esta.

\section{Referencias bibliográficas}

$\mathrm{Wu}$ Y, Liao L, Lin F. The diagnostic protocol for hereditary spherocytosis-2021 update. J Clin Lab Anal [Internet]. 2021 Dec 1 [cited 2022 Jan 5];35(12). Available from: /pmc/articles/PMC8649336/

Ciepiela O. Old and new insights into the diagnosis of hereditary spherocytosis. Ann Transl Med [Internet]. 2018 Sep [cited 2021 Dec 16];6(17):339-339. Available from: /pmc/articles/PMC6174190/

Haley K. Congenital Hemolytic Anemia. Med Clin North Am [Internet]. 2017 Mar 1 [cited 2021 Dec 16];101(2):361-74. Available from: http://www.revhematologia.sld.cu/index.php/hih/article/view/1098/1017

Liao L, Xu Y, Wei H, Qiu Y, Chen W, Huang J, et al. Blood cell parameters for screening and diagnosis of hereditary spherocytosis. J Clin Lab Anal [Internet]. 2019 May 1 [cited 2021 Dec 16];33(4). Available from: /pmc/articles/PMC6528600/ 
Wang X, Zhang A, Huang M, Chen L, Hu Q, Lu Y, et al. Genetic and Clinical Characteristics of Patients with Hereditary Spherocytosis in Hubei Province of China. Front Genet [Internet]. 2020 Aug 18 [cited 2021 Dec 16]; 11:953. Available from: /pmc/articles/PMC7461774/

Medica R, Rica DEC, Lxxiv C. Esferocitosis Hereditaria. Rev Medica Costa Rica y Centroam. 2017;(622):19-23.

Zamora EA, Schaefer CA. Hereditary Spherocytosis. In: StatPearls. Treasure Island (FL); 2021.

Donato H, Nacional De Hematología C, Renée D, Crisp L, María D, Rapetti C, et al. Esferocitosis hereditaria. Revisión. Parte II. Manifestaciones clínicas, evolución, complicaciones y tratamiento. Arch Argent Pediatr [Internet]. 2019 [cited 2021 Dec 16];113(2):168-76. Available from: http://dx.doi.org/10.5546/aap.2015.168

Crisp RL, García E, Solari L, Rapetti MA, Nesse AB, Donato H. Esferocitosis hereditaria: experiencia clínica y diagnóstica en Argentina. Hematología [Internet]. 2019 [cited 2021 Dec 16]; Available from: https://ri.conicet.gov.ar/handle/11336/84692

Ates U, Tastekin NY, Gollu G, Ergun E, Yagmurlu A. Esplenectomía laparoscópica en la población pediátrica: seguimiento a largo plazo. Arch argent pediatr [Internet]. 2017 Dec 1 [cited 2021 Dec 16];115(6):385-90. Available from: https://www.sap.org.ar/docs/publicaciones/archivosarg/2017/v115n6a14.pdf

Okamoto T, Shima H, Noma Y, Komatsu M, Azuma H, Miya K, et al. Hereditary spherocytosis diagnosed with extremely low glycated hemoglobin compared to plasma glucose levels. Diabetol Int [Internet]. 2021 Apr 1 [cited 2022 Jan 5];12(2):229. Available from:/pmc/articles/PMC7943674/

Rodríguez JLA, Ulloa JMA, Alvarado WSA, Llanos MSN, Alquizar CAGA. Anemia Hemolítica en Esferocitosis Hereditaria: Reporte de Caso. Rev. Médica Trujillo [Internet]. 2018 Apr 2 [cited 2021 Dec 16];13(1). Available from: https://revistas.unitru.edu.pe/index.php/RMT/article/view/1753

Güngör A, Yaralı N, Fettah A, Ok-Bozkaya İ, Özbek N, Kara A. Hereditary spherocytosis: Retrospective evaluation of 65 children. Turk J Pediatr. 2018;60(3):264-9.

Manciu S, Matei E, Trandafir B. Hereditary spherocytosis - diagnosis, surgical treatment and outcomes. A literature reviews. Chir. 2017 Mar 1;112(2):110-6. 


\section{ADÉnigital}

De H, De C, De Titulación T. Análisis clínico y molecular de una paciente de 19 años con diagnóstico de Esferocitosis Hereditaria. Univ. San Fr Quito USFQ Col Ciencias La Salud. 2019;

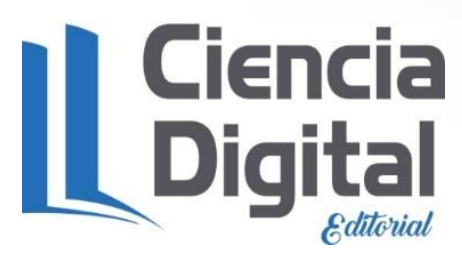


El artículo que se publica es de exclusiva responsabilidad de los autores y no necesariamente reflejan el pensamiento de la Revista Anatomía Digital.

\section{\Ciencia}

El artículo queda en propiedad de la revista y, por tanto, su publicación parcial y/o total en otro medio tiene que ser autorizado por el director de la Revista Anatomía Digital.
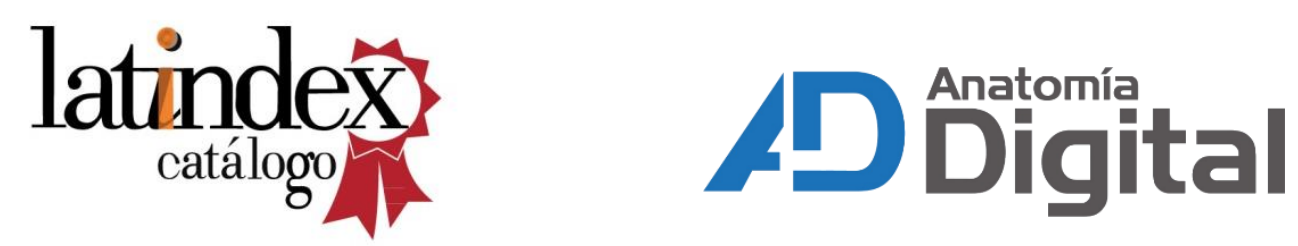

Indexaciones

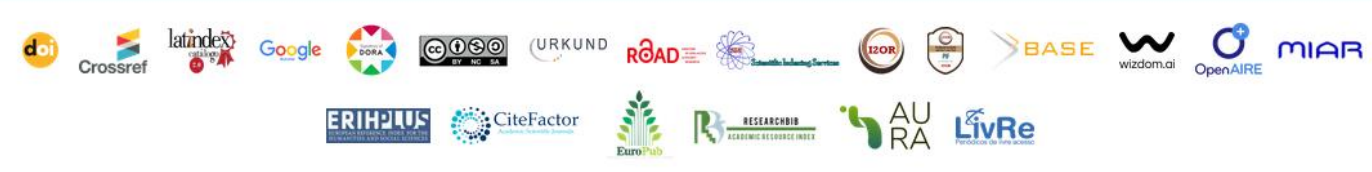

